\title{
Creatividad, complejidad y formación: un enfoque transdisciplinar
}

\author{
Creativity, complexity and training: \\ A transdisciplinary approach \\ Jessica CABRERA CUEVAS y Agustín de la HERRÁN GASCÓN \\ Universidad Autónoma de Madrid
}

Recibido: Enero 2014

Aceptado: Julio 2014

\begin{abstract}
Resumen
La presente investigación se desarrolló en el contexto de la formación universitaria. Se centra en las aplicaciones de la creatividad desde una visión transdisciplinar y de complejidad evolutiva. Una de las intenciones del estudio es explorar el fenómeno de la creatividad desde la complejidad en la práctica docente, con el fin de obtener una aproximación general al modo en que se comprende y aplica por expertos que la investigan y enseñan. El objetivo específico se refiere a identificar las tendencias en la aplicación didáctica de la creatividad en el contexto de la enseñanza universitaria. La metodología utilizada se basó en la complementariedad paradigmática (Hashimoto, 2013), con entrevistas en profundidad y encuesta a docentesinvestigadores de creatividad de 14 países. Los instrumentos y técnicas utilizadas han sido registros de observación, cuestionario validado, notas de campo, soporte para registro en audio, validación de instrumentos y triangulación. Los resultados más destacados del objetivo de la investigación llevan a concluir, según las tendencias más recientes en epistemología de la creatividad, con que la transdisciplinariedad y el enfoque de complejidad evolutiva son potentes referentes metodológicos y prácticos para las múltiples aplicaciones en la enseñanza universitaria de la creatividad. Las conclusiones giran en torno a algunas características que parece ir adoptando la enseñanza y la formación en creatividad universitaria, globalmente asimilables a los enfoques transdisciplinares y con base en la complejidad y la conciencia.
\end{abstract}

Palabras clave: creatividad, formación, complejidad, transdisciplinariedad.

\begin{abstract}
This research was conducted in the context of university education. It focuses on the application of creativity from a transdisciplinary vision of evolutionary complexity. One of the aims of the study was to explore the phenomenon of creativity from the perspective of complexity in teaching, in order to obtain a general perception of how this concept is understood and applied by experts who investigate and teach it. The specific aim is to identify trends in the didactic application of creativity in the context of university education. The methodology used was based on paradigmatic complementarity (Hashimoto, 2013), with in-depth interviews and surveys administered to faculty researchers of creativity from 14 countries. The tools and


techniques used were observation records, validated questionnaires, field notes, audio support or registration, validation of instruments and triangulation. The most important results of the study lead to the conclusion that, according to the latest trends in epistemology of creativity, the transdisciplinary approach and evolutionary complexity are powerful methodological and practical references for multiple applications in teaching creativity at the university level. The findings hinge around some features that education and college creativity training seem to be adopting, globally comparable to the cross-disciplinary approaches and based on the complexity and consciousness.

Keywords: creativity, training, complexity, transdisciplinarity.

Desde el año 2007 se desarrolla una investigación para profundizar en el campo de estudio de la creatividad. Al acercarnos a los modelos más consensuados que explican su conocimiento y aplicación, nos encontramos con diferentes propuestas que categorizan el fenómeno parcialmente o con dimensiones separadas. Destacamos la aproximación psicodinámica (Freud, 1923; Kris, 1952; Kubie, 1958), la división dual de la creatividad en personal e histórica (Boden, 1994) -redefinida por Herrán (1998) en personal, sobresaliente (novedosa para un grupo relativo de referencia) e histórica-, la creatividad desde la pragmática (De Bono, 1977), desde la psicometría (Guilford, 1950), desde el enfoque humanista (Maslow,1985; Rogers, 1980; Blay, 1980), desde una visión de confluencia o componencial (Amabile, 1983; Sternberg, 1977; Csikszentmihalyi, 1996), y desde el enfoque más usado: la división de persona, proceso, producto y ambiente (Mooney, 1957; MacKinnon, 1975; Runko, 2007; Landau, 2002; Torre, 2006, entre otros) (en Cabrera, 2008, 2011, adaptado).

En opinión de algunos investigadores (Romo, 1997; Landau, 2002; López Pérez, 2006; Alfonso, 2006) los modelos componenciales conocidos aún son insuficientes para abarcar todo el fenómeno de la creatividad. Por ello, consideramos de especial relevancia el movimiento más emergentes en torno a la complejidad y transdisciplinariedad, las aportaciones realizadas desde España desde el enfoque transdisciplinar y ecosistémico (Torre, 2007) y el complejo-evolucionista o radical e inclusivo (de la Herrán, 2003, 2005, 2014).

Consideramos necesario otorgar las evidencias que den cuenta de la relevancia social de la creatividad desde una visión compleja y transdisciplinar en todos los ámbitos. La perspectiva de quienes investigan y enseñan creatividad puede ser un valioso aporte para su aplicación social.

\section{Marco teórico}

\section{Creatividad y complejidad evolutiva}

El estudio de la creatividad ha ido incluyendo a lo largo del tiempo diferentes dimensiones de profundización. Primero hubo una creatividad centrada en un tipo particular de individuos, luego con una fuerte carga psicológica se asoció a variables aisladas y a eficiencia. Los métodos creativos tenían una clara orientación instrumental 
asociada a la solución de problemas en el mundo del trabajo, pero sin considerar aún por ejemplo, las relaciones interpersonales. Fue necesaria una visión más amplia y contextualizadora que integrara lo sistémico. Sin embargo, hoy la creatividad ya no queda encasillada en ninguno de ellos, ha trascendido las fronteras disciplinares de sus procesos cognitivos, estilos de pensamiento, personalidad, de sus evaluaciones de productos, etc. Ya no nos podemos quedar limitados a los viejos y repetidos usos. La creatividad, como plantea de la Herrán (2009a, 2009b, 2010) no puede definirse desde lecturas parciales del fenómeno creativo, como habitualmente y en marcos científicos se hace, bien como sensibilidad para mirar de forma diferente, redefinición, capacidad para descubrir o resolver problemas, con divergencia de pensamiento, con generación de ideas, con pensamiento lateral, imaginación, creación o producto, imaginación, innovación, etc. Hacerlo es incurrir en un error de pars pro toto que según el autor continúa, al parecer, poco advertido entre la comunidad científica o experta ocupada en estudiar la creatividad.

La complejidad es una realidad, como lo es que en toda manifestación humana intervienen los pensamientos, las emociones, el lenguaje, la corporalidad de manera simultánea, complejo, a decir de Morin (2004), significa aquello que forma un tejido conjunto. Es importante considerar también, los aspectos valóricos y de evolución de conciencia en todos los niveles: personal, social, ecológico y universal (Cabrera, 2009). Si en los campos de interacciones humanas se da de manera natural la complejidad de redes personales, sociales y ecológicas ¿cómo vamos a formar en creatividad desde lo parcial y descontextualizado? Trabajar la creatividad desde una mirada transdisciplinar y ecosistémica supone considerarla integrando todos los aportes hasta ahora estudiados desde diferentes disciplinas (psicología, pedagogía, filosofía, sociología); la nuevas aportaciones científicas (neurociencia, neurocardiología, física cuántica) y más aún, considerando otros campos del saber que enriquezcan su aplicación y de los cuales ya están dando cuenta nuevas investigaciones. Por otra parte, la complejidad como elemento emergente en los estudios sobre creatividad, no sería condición suficiente para distinguir la creatividad como "un adjetivo de" (las personas, los procesos, los productos y los ambientes) como tanto se ha insistido en los modelos consensuados. Si bien esto pudiera tener una complejidad creciente en sí mismo -en ese mismo orden-, se han considerado como pilares separados entre sí. En todo el recorrido, los estudios sobre creatividad continúan en evolución cada vez con mayor complejidad, es decir, integrando nuevos elementos.

Las propuestas que emergen desde el paradigma de la complejidad, el enfoque transdisciplinar y el enfoque complejo evolucionista desde donde se sitúan los autores, se basan en una amplia diversidad de reconocidos científicos e investigadores que, desde sus diferentes referentes tanto de conocimiento, como de las tradiciones relativas al desarrollo de la salud, la sabiduría y la conciencia entre otros, aportan al campo de la creatividad. Algunos de estas aportaciones desde una perspectiva de complejidad las encontramos en el enfoque transpersonal de la conciencia (Wilber, 2005; Ferrer, 2003; Rubia Vila, 2007; Almendro, 2006; González-Garza, 2006), el enfoque complejo de Morin (2004); el enfoque ecosistémico y de complejidad de Moraes (2007) y Torre y Moraes (2006, 2008), la teoría interactiva y psicosocial (e (2006), la teoría complejo evolucionista o radical e inclusiva, de Herrán (2003, 2005, 2014), entre otros. 
Llegamos a un punto donde en creatividad se asocia por tanto, a la complejidad, la trascendencia, la espiritualidad y la evolución humana basada en la conciencia. No podemos dejar de vincular, pues esta visión, tanto para quien la estudia, enseña e investiga, como para el artista, profesional o científico que la aplica, para cada persona que la vive en diferentes intensidades y niveles de conciencia, como para el contexto que la recibe, y comparte, ya sea con un otro, un sistema de otros, la sociedad o la naturaleza.

\section{Creatividad y transdisciplinariedad}

En el primer Ciclo de Conferencias sobre Complejidad y Modelo Pedagógico organizado por el Centro UNESCO de la comunidad de Madrid en 2008 y 2009, se discutieron diversas temáticas en relación con la problemática educativa actual y los nuevos desafíos sociales que ello implica. Torre (2010) señala que aún hay mucho que hacer en temas como la desmotivación, la violencia, el estrés, la fragmentación del conocimiento, el academicismo informativo, la pérdida de valores y la educación alejada de la vida, lo social y ambiental. Sin duda el escenario define una realidad educativa a la que intentan dar respuestas algunos investigadores como Morin (2003), Herrán (2009a, 2009b, 2011b, 2014) y de la Torre (2010) entre otros. Para ello, la visión pedagógica que se pretende desde el nuevo paradigma transdisciplinar y complejo es que las instituciones educativas sean un espacio de transformación personal, institucional y social, tanto en lo cultural y científico, como en los valores humanos, éticos y espirituales basados en la confianza, en la cooperación, en la creatividad y en el reconocimiento. Un lugar donde se potencie lo mejor de cada persona desde su formación. Desde el planteamiento transdisciplinar se propone que la realidad ya no puede ser abordada desde la fragmentación disciplinar, sino desde la complejidad, desde los conocimientos que se generan entre, a través de y más allá de las disciplinas. Por tanto, el paradigma educativo transdisciplinar ha de ser humanista, social, ecológico y sostenible. Una mirada de la investigación y de la formación basada en valores humanos, en el desarrollo de la conciencia, en la creación de redes y campos de emprendizaje. Lo transdisciplinar no se identifica con teorías, paradigmas o modelos, sino con una manera de mirar la realidad y sus diferentes niveles fruto de la percepción y de la conciencia (Torre, Pujol y Sanz, 2007). Herrán (2011a, 2013) va más allá e identifica lo transdisciplinar como una cualidad del conocimiento, susceptible por tanto de mal uso o de estafa epistemológica por quienes lo entienden y manejan como algo diferente o como un añadido al propio acto de conocer o del proceder natural de la razón, intrínsecamente complejo.

Considerando estos referentes vinculables al ámbito de la formación en creatividad, podemos observar que ésta requiere una apuesta integral y compleja. Una creatividad que considere diferentes disciplinas científicas, diferentes recursos materiales y tecnológicos, diferentes formas de expresión y diferentes respuestas a las constantes emergencias del contexto, y sobre todo, una creatividad que responda a cuestiones que afectan no sólo a la persona, sino, como señala Moraes (2007), a una prioridad educativa que incluye a la ciudadanía planetaria, la sustentabilidad ecológica y a la evolución de la conciencia humana. Lo nuevo siempre trae consigo actitudes de duda, 
temor a los cambios y en algunas ocasiones, muchas resistencias. El cambio en las personas sin duda es mucho más lento que el avance de las nuevas tecnologías. Sin embargo, podemos observar que el poder de la intención, la sintonía de diferentes personas y colectivos sociales vinculados a los valores humanos, la colaboración y el trabajo en red, así como las evidencias de muchos docentes e investigadores de la creatividad comprometidos con la complejidad y la innovación, nos invitan a sumar sinergias para favorecer los cambios sociales y ecosistémicos en beneficio del bien común y de la evolución humana.

Modelo de tendencias en enfoques de creatividad desde una conciencia compleja-evolutiva

Desde una visión de complejidad evolutiva (Cabrera, 2008, 2009, 2011) y el paradigma de la complejidad, proponemos un modelo teórico sobre los enfoques del estudio de la creatividad (Herrán, 2008). Su utilidad contextualiza la visión de los docentes investigadores entrevistados y facilita y orienta los análisis de datos y resultados. El modelo presenta una primera clasificación de los enfoques en creatividad, desde los más conocidos a los más emergentes, en que se sitúan los autores de este artículo. Se dividen estos dos criterios y en cuatro niveles de creciente complejidad en las categorías referidas a: un tipo de individuos, a todas las personas, al sistema y a la complejidad. Con un tercer criterio se plantea asociar las teorías y enfoques a aquellos investigadores que centran la creatividad desde: los impulsos, la medición, la estimulación, la evaluación, el desarrollo humano así como desde la confluencia y la transdisciplinariedad. Esta topología se elaboró a partir de fuentes primarias y secundarias y por expertos representativos de los enfoques en más de una de ellas a la vez (Figura 1). 


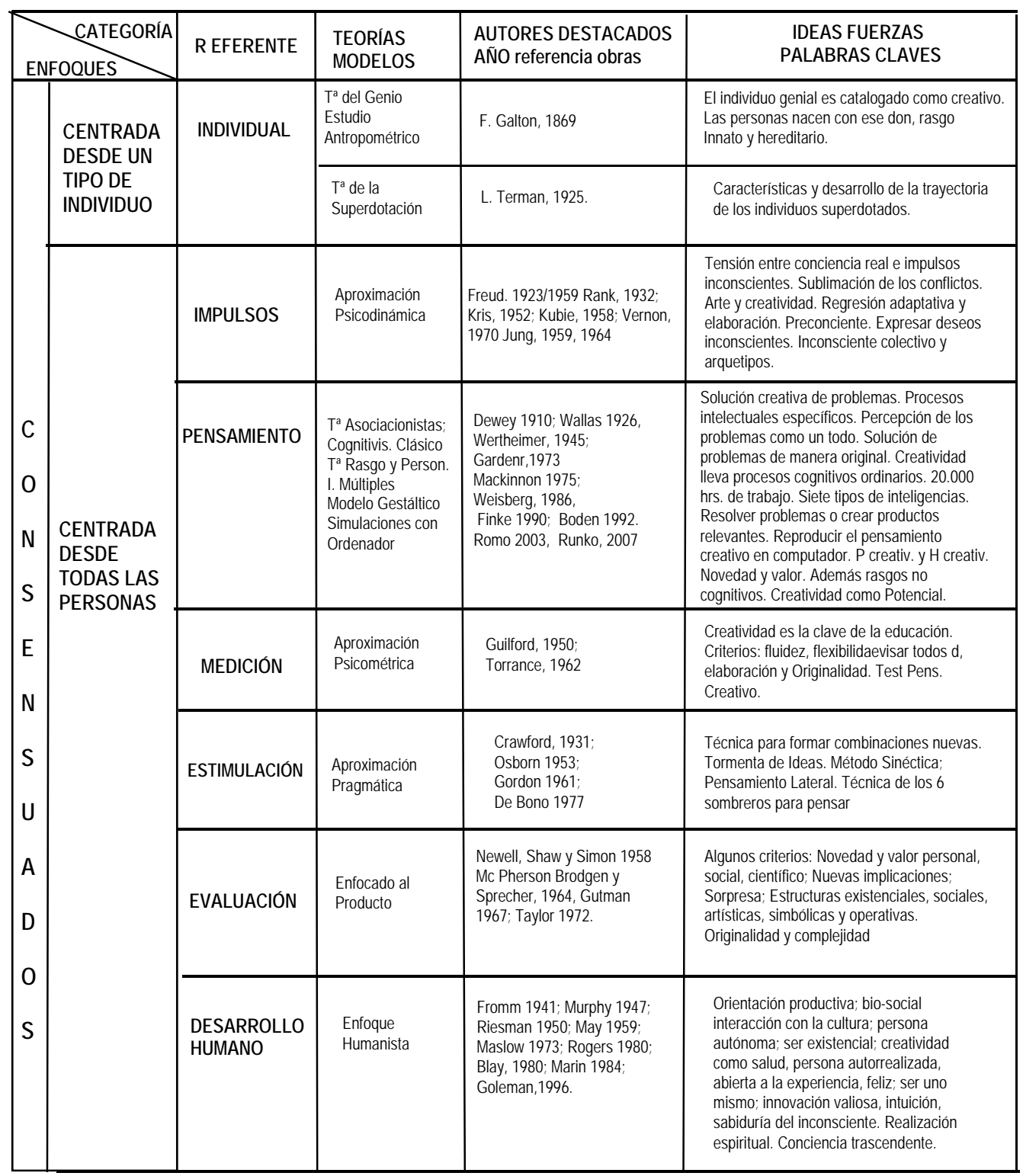

Figura 1. Modelo de Tendencias en Enfoques sobre Creatividad cCC

Fuente: Cabrera, J. (2011) 


\begin{tabular}{|c|c|c|c|c|c|}
\hline \multicolumn{2}{|c|}{ CATEGORÍA } & REFERENTE & $\begin{array}{l}\text { TEORÍAS } \\
\text { MODELOS }\end{array}$ & $\begin{array}{l}\text { AUTORES DESTACADOS } \\
\text { AÑO referencia obras }\end{array}$ & \multirow{2}{*}{\begin{tabular}{l}
\multicolumn{1}{c}{$\begin{array}{l}\text { IDEAS PUERZAS } \\
\text { PALABRASCLAVES }\end{array}$} \\
Creación científica como resultado de una vida \\
de trabajo. Producciones creadoras como \\
variaciones de ajuste adaptativo. Creatividad \\
necesita articulación psicosocial, individual y \\
social. Futuro y creatividad con mirada \\
prospectiva social.
\end{tabular}} \\
\hline \multirow{5}{*}{$\begin{array}{l}C \\
O \\
N \\
S \\
E \\
N \\
S \\
U \\
A \\
D \\
O \\
S\end{array}$} & \multirow{5}{*}{$\begin{array}{l}\text { CENIRADA } \\
\text { DESDE EL } \\
\text { SISTEMA }\end{array}$} & \multirow{5}{*}{$\begin{array}{c}\text { DE } \\
\text { CONFLUENCIA }\end{array}$} & Ta Socialcultural & $\begin{array}{l}\text { H. Gruber, } 1974 \\
\text { Simonton, } 1981 \\
\text { Rodríauez }\end{array}$ & \\
\hline & & & $\begin{array}{l}\text { Ta social. Modelo } \\
\text { Componencial }\end{array}$ & $\begin{array}{l}\text { T. Amabile, } \\
1983 \text { a la Act. }\end{array}$ & $\begin{array}{l}\text { Integra diferentes procesos; destaca el } \\
\text { ambiente Sociocultural, competencias } \\
\text { personales, creativas, Motivación intrínseca. }\end{array}$ \\
\hline & & & Ta de la Inversión & $\begin{array}{l}\text { R. Sternberg, } \\
1977 \text { a la Act. }\end{array}$ & $\begin{array}{l}\text { Integra } 6 \text { recursos: Habilidades intelectuales, } \\
\text { conocimiento, estilos de pensamiento, } \\
\text { personalidad, motivación, ambiente. }\end{array}$ \\
\hline & & & Ta Ecológica & $\begin{array}{l}\text { M. Csikszentmihalyi, } \\
1996 \text { a la Act. }\end{array}$ & $\begin{array}{l}\text { Destaca el medio histórico y social de las obras } \\
\text { creativas. Estado de fluir como alto nivel de } \\
\text { creatividad. Individuo - Campo - Ámbito. }\end{array}$ \\
\hline & & & $\begin{array}{l}\text { Ta Creatividad } \text { Cricada Total } \\
\text { Aplo }\end{array}$ & $\begin{array}{l}\text { D. de Prado } \\
1988 \text { a la Act. }\end{array}$ & $\begin{array}{l}\text { Tecnocreática socio humanística. Visión } \\
\text { humanística integral de la creatividad en la } \\
\text { práctica. Multilenguajes. Didáctica creativa } \\
\text { autoconsciente. }\end{array}$ \\
\hline & \multirow{4}{*}{$\begin{array}{l}\text { CENIRADA } \\
\text { DESDELA } \\
\text { COMPLE- } \\
\text { JIDAD }\end{array}$} & \multirow{4}{*}{$\begin{array}{l}\text { TRANSDIS } \\
\text { CIPUNAR }\end{array}$} & \multirow{2}{*}{$\begin{array}{l}\mathrm{T}^{\mathrm{a}} \text { Interactiva y } \\
\text { Psicosocial. }\end{array}$} & S. de la Torre, 1982 a la Act. & \multirow{2}{*}{$\begin{array}{l}\text { Saber educar en la complejidad de la era } \\
\text { planetaria. Sustentabilidad ecológica. } \\
\text { Paradigma ecosistémico. Creatividad como } \\
\text { parte de un todo personal, social y cósmico que } \\
\text { se manifiesta como flujos de energía. Integra } \\
\text { emoción, pensamiento y acción. Carácter } \\
\text { dinámico, interactivo, sistémico y complejo. } \\
\text { Creatividad más que generación de ideas, } \\
\text { como campos de vibración. Creatividad } \\
\text { cuántica. Formar en creatividad es apostar por } \\
\text { Un futuro de progreso, de justicia, de tolerancia } \\
\text { y de convivencia. }\end{array}$} \\
\hline $\begin{array}{l}\mathbf{E} \\
\mathbf{M} \\
\mathbf{E} \\
\mathbf{R} \\
\mathbf{G} \\
\mathbf{E} \\
\mathbf{N}\end{array}$ & & & & $\begin{array}{l}\text { AUTORES DE REFERENCIA } \\
\text { Morin; } 1981 \text { a la Act. } \\
\text { Maturana, Varela, Binnig, Laszlo, } \\
\text { Lorenz, Prigogine, Moraes, } \\
\text { Capra, Briggs, Pribram, Wilber, } \\
\text { Bohm, Roger Ciurana, } \\
\text { D'Ambrosio, Nicolescu, Zukav, } \\
\text { May, Damasio, Lipton, } \\
\text { Servan-Schreiber, Otros. }\end{array}$ & \\
\hline $\begin{array}{l}\mathrm{T} \\
\mathrm{E} \\
\mathbf{C}\end{array}$ & & & \multirow{2}{*}{$\begin{array}{l}\text { Enfoque } \\
\text { Complejo } \\
\text { Evolucionista o } \\
\text { radical e inclusivo }\end{array}$} & A. de la Herrán, 1998 - a la Act. & \multirow{2}{*}{$\begin{array}{l}\text { Constructos: egocentrismo, complejidad de } \\
\text { conciencia, conocimiento, noosfera, } \\
\text { evolución humana, universalidad, } \\
\text { humanidad, muerte, creatividad total, } \\
\text { enfoque radical e inclusivo de la formación, } \\
\text { etc. Déficit radical de la educación y } \\
\text { formación. Posibilidad de inclusión curricular } \\
\text { de temas o retos radicales. Es preciso } \\
\text { profundizar más allá del ego, de los ismos, } \\
\text { de la periferia. La creatividad total tiene tres } \\
\text { destinos: el crecimiento personal, la mejora } \\
\text { social y la evolución de la conciencia. En } \\
\text { educación la creatividad es secundaria. }\end{array}$} \\
\hline $\mathrm{s}$ & & & & $\begin{array}{l}\text { AUTORES DE REFERENCIA } \\
\text { Lao Tse, Confucio, Buda, } \\
\text { Sócrates, Zhuang zi, Kant, } \\
\text { Herder, Hegel, Fröebel, Martí, } \\
\text { Eucken, Nietzsche, Teilhard de } \\
\text { Chardin, Montessori, Maslow, } \\
\text { Dürckheim, Krishnamurti, } \\
\text { Deshimaru, Fromm, Blay, Morin, } \\
\text { García-Bermejo, González- } \\
\text { Jiménez, Osho, cada persona... }\end{array}$ & \\
\hline
\end{tabular}

Figura 1. Continuación. Modelo de Tendencias en Enfoques sobre Creatividad cCC

Esta propuesta, en la que se intenta elaborar una organización integral de los enfoques en creatividad, en ningún caso se plantea reflejar la totalidad del campo epistemológico de la creatividad, sino más bien y en consonancia con su naturaleza, 
deducir una síntesis con algún valor didáctico para la formación e investigación en creatividad.

\section{Metodología de investigación}

Se presenta un cuestionamiento inicial del origen del problema para llegar a plantearnos en qué medida se consideran los atributos transdisciplinares y de complejidad en la formación en creatividad. Para ello presentamos una justificación interna de la motivación por este tema y una justificación externa relacionada con la fragmentación del conocimiento en creatividad desde los modelos conocidos.

El procedimiento de la investigación se dividió en tres fases que se fueron retroalimentando mutuamente durante el estudio. En primer lugar se realizó una revisión de los estudios sobre creatividad y se construye una propuesta de síntesis de sus enfoques con una tendencia compleja-evolutiva validada por expertos y llegando a tener veintidós revisiones. Luego se realizó una estancia y trabajo de campo para profundizar en la visión transdisciplinar, facilitada por el Grupo de Investigación y Asesoramiento Didáctico GIAD, de la UB (Cabrera, 2010). Finalmente se realizaron otras validaciones a los instrumentos aplicados hasta llegar a una prueba piloto y su aplicación final.

Los supuestos del estudio señalan que las tendencias en la comprensión y aplicación de la creatividad se orientan a crecientes niveles de complejidad y transdisciplinariedad. Por tanto la pregunta orientadora que guía el estudio es ¿desde qué enfoque se aplica la creatividad?

El objetivo específico que nos ocupa en el presente artículo es identificar las tendencias en la aplicación de la creatividad en la formación universitaria en la actualidad. Por la naturaleza compleja de la creatividad, el estudio compone un diseño metodológico que destaca nuestra visión epistemológica que va de la mano de la transdisciplinariedad y complejidad evolutiva integrando diferentes enfoques epistemológicos considerando principalmente el paradigma de la complejidad evolutiva o radical e inclusivo (Herrán, 2003, 2005, 2014) como eje transversal en su dimensión teórico-práctica. Es por tanto integrador y abierto, por cuanto admite combinar atributos de diferentes enfoques para adaptarse y resolver lo mejor posible los problemas de investigación (Herrán, 2005:453-454). Pero además es ecléctico, basado en la complementariedad paradigmática definida por Hashimoto (2013), por cuanto integra no sólo lo cualitativo y lo cuantitativo, sino la percepción de los paradigmas establecidos (positivista, interpretativo y teórico crítico) acerca del mismo objeto de estudio.

Al respecto nos planteamos una metodología mixta o integradora con técnicas e instrumentos cuantitativos y cualitativos que permitieron un trabajo de campo minucioso, así como el desarrollo de entrevistas a expertas/os en creatividad de catorce países.

El instrumento elaborado para las entrevistas supone un trabajo de revisión teórica desarrollada durante dos años y un proceso de validación que asocia credibilidad a nuestros resultados que serán analizados con programas cuantitativos y cualitativos. 
En lo que respecta a estrategias hemos ido transitando por varios métodos. Utilizamos como expresan estrategias interactivas Mc Millan y Schumacher (2005:401), utilizando observación participante, entrevistas en profundidad, observación directa, aplicación de encuestas, etc. lo cual da cuenta de las decisiones de proceso que se fueron concretando en la investigación.

El esfuerzo final para contactar con investigadores y docentes expertos en creatividad de diferentes países ha hecho posible alcanzar la cifra de cuarenta y cuatro participantes, que supera el $50 \%$ del grupo inicialmente contactado. Los aceptantes expresaron muy buena disposición a participar. La cantidad puede considerarse cercana a la óptima, basándonos en la horquilla entre 20 y 50 propuesta por Mc Millan y Schumacher (2005:144) para la investigación con grupos menores. Los agrupamos en las siguientes categorías:

- $\quad$ Tipo de experto: Docente en investigador en creatividad $=32$

- $\quad$ Docente en creatividad $=11$

- Investigador en creatividad $=1$

- Género: Masculino = 26

- Femenino $=18$

- Área: Pedagogía = 16

- Psicología = 11

- Otras disciplinas $=10$

- Bellas Artes $=7$

- $\quad$ Tipo de relación establecida Personal $=33$

- $\quad$ Virtual = 11

- Por países: España:19; Argentina: 6; México:4; Chile:3; Brasil:2; Colombia: 2; Portugal:1; Canadá:1; Guatemala:1; Perú:1; Venezuela:1; Inglaterra:1; Estados Unidos:1; Cuba:1.

- Total: 44.

Las técnicas utilizadas en el estudio fueron: Análisis documental, observación, trabajo de campo, entrevistas, Grabación de audio, así como la participación en diecinueve eventos pertinentes que hicieron las veces de fuentes de producción, recogida y contraste de datos vinculados al tema de la investigación (Mc Millan y Schumacher, 2005:454) los cuales influyeron en la fundamentación, validación, profundización, cohesión y reorientación del proceso investigativo, como también resultaron importantes para el diálogo y reuniones con expertos, establecimiento de contactos y en alguna ocasión para la celebración de entrevistas

Para recoger información relevante para el estudio se emplearon los siguientes instrumentos y técnicas asociadas: Pautas de registros de observación (elaboradas a partir del trabajo del grupo de investigación GIAD de la Universidad de Barcelona utilizadas durante las observaciones etnográficas en el aula en dicha universidad, donde se profundizó en la utilidad de enfoque transdisciplinar y elaborado una síntesis de los atributos transdisciplinares en la formación universitaria); cuestionario de la entrevista (cuyo contenido fue validado por expertos en metodología, en creatividad y aplicado en prueba piloto. Está compuesto por dos apartados bien definidos: Las 
preguntas orientadoras de los temas según los objetivos, y la encuesta. El tipo de cuestionario es semiestructurado, permitiendo respuestas individuales pero específicas en su intención (Mc Millan y Schumacher, 2005:250); el instrumento de la encuesta su elaboración requirió dos años y comprendió la profundización en los enfoques de creatividad y una aproximación a la síntesis de dichos enfoques y teorías con ideas fuerza deducidas de ellos e inducidas del análisis sus estudios más relevantes-. Todo su proceso de elaboración fue contrastado con expertos en creatividad que además eran buenos conocedores de su teoría y de su historia. El criterio del orden utilizado en los ítems referidos a las ideas fuerza de los enfoques, como al de los nombres de dichas teorías fue intencionadamente aleatorio); notas de campo (han sido un soporte relevante en todo el proceso, con innumerables cuadernos, libretas, registros de audio, observaciones de contexto, y toma de imágenes que han servido para tejer el entramado del estudio); soporte y registro de audio (para transcripciones literales de las entrevistas); depuración y validación de instrumentos (contraste del diseño general con dos expertos en metodología de la investigación y con otros expertos en creatividad para contrastar sobre todo, los resúmenes asociados a las 20 teorías y enfoques, quienes aportaron sus consideraciones para su posterior validación en la prueba piloto. Se estuvo trabajando en la elaboración de diseño del instrumento, con anotaciones de metatextos y versiones de mejoras las cuales llegó a su versión final número 22) y la triangulación (para nuestro estudio, cuando ha sido posible, los mismos datos se han considerado desde una amplia variedad de personas y de técnicas, lo que ha permitido confirmar datos y aumentar la credibilidad de los resultados).

\section{Resultados}

Para el estudio que nos ocupa, analizamos los ítems de cada entrevista correspondientes a la encuesta (utilizando SPSS) y a las respuestas abiertas de los temas presentados (Programa QDA Miner) (Sanchez-Criado y Blanco, 2005). Así se fueron generando las posibilidades de codificación. Es preciso destacar que la codificación se efectuó una vez recogido todos los datos, y fuimos elaborando las características relevantes de los testimonios para transformarlas en unidades que permitieran su descripción o análisis más preciso (Hernández Sampieri, Fernández Collado y Baptista Lucio, 2003:303). Hemos de confesar que en algunos casos, resultó muy difícil encontrar una unidad en la que poder codificar, y en más de un caso, nos sirvió nuestro propia mirada para el "Modelo de tendencias en creatividad cCC" para organizar los contenidos, considerando tópicos que van desde los cognitivo, a lo humanista, lo sistémico y lo complejo, pero reiteramos, no fueron códigos que se hubieran pensado con anterioridad, sino que resultaron como mayormente adecuados para el análisis.

En el caso concreto de los enfoques de creatividad existentes, se pidió a las/os expertas/os valorar su grado de acuerdo con ocho clasificaciones que se han realizado como propuestas que reflejan el campo de la creatividad, entendiendo por campo, el conocimiento y las aplicaciones de la creatividad, mas sin indicar el nombre de los mismos. El resultado para el Modelo de Tendencias en creatividad cCC, tuvo una alta 
puntuación, sumando 41 expertos (de 42) que están entre Bastante y Totalmente de acuerdo con esta forma de representar el campo de la creatividad (Gráfico 1).

\section{Modelo de tendencias en estudio de Creatividad}

(\%) $n=42$

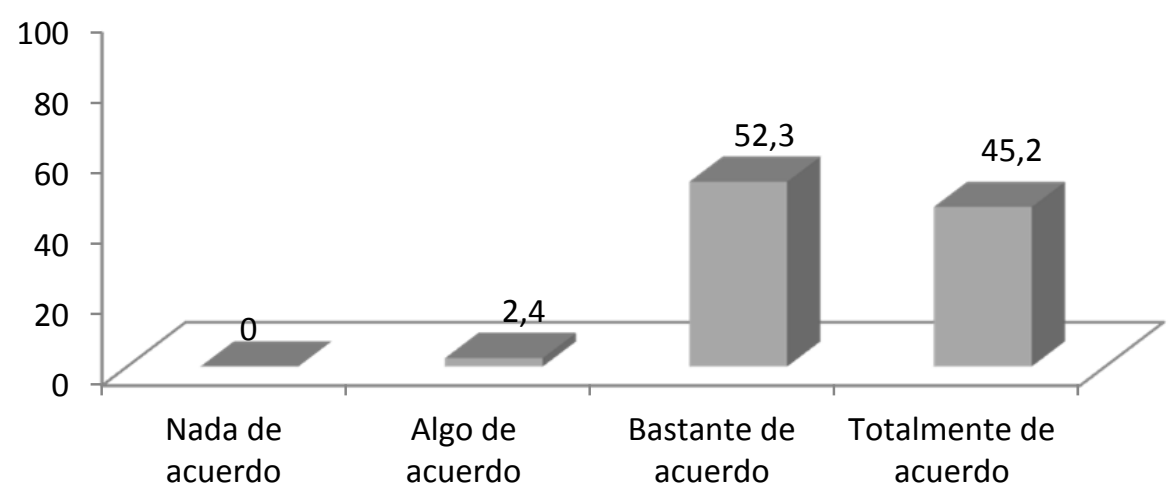

Gráfico 1 Modelo de tendencias en estudio sobre creatividad.

Fuente: Elaboración propia

Las expertas/os entrevistadas/os han manifestado también la necesidad de integrar los conocimientos sobre creatividad, considerando nuestro estudio como una propuesta que hace reflexionar, que aporta y que puede servir de referencia para la formación en creatividad.

Tras el trabajo de campo con el Grupo GIAD de la Universidad de Barcelona -donde se realizó la indagación respecto de la pertinencia del enfoque transdisciplinar en la enseñanza universitaria- se obtuvieron los siguientes resultados:

- La práctica docente con visión transdisciplinar favorece la motivación, el trabajo colaborativo, el respeto, la libertad de expresión y el compromiso social.

- El enfoque transdisciplinar se sustenta en principios de mejora personal, formativa y con una relación ecológica sustentable que se ve promovida por las/os docentes que se comprometen con esta propuesta educativa y social.

En la aplicación de este enfoque, preguntamos acerca de los atributos transdisciplinares para la práctica de la creatividad en la formación universitaria, (visión y planificación, rol docente, rol discente, clima, organización, estrategias y recursos, actividades y evaluación) encontrando sobre un 90 \% entre bastante y totalmente de acuerdo sobre los mismos. 
Por otra parte, un interesante resultado nos arrojó el comparar el grado de conocimiento de las teorías y enfoques con su grado de acuerdo sobre los mismos (Gráfico 2). Puede observarse que los expertos en creatividad participantes afirman conocer y aplicar mejor las teorías más cognitivas o de enfoque humanista que las emergentes asimilables a la complejidad, sin embargo, su forma de comprender la creatividad está orientada a lo sistémico y de complejidad. Es decir, existe una tendencia de la comprensión de los enfoques en creatividad, hacia mayores niveles de complejidad.

\section{Grado de conocimiento/acuerdo con enfoques de creatividad}

(\%) $n=42$
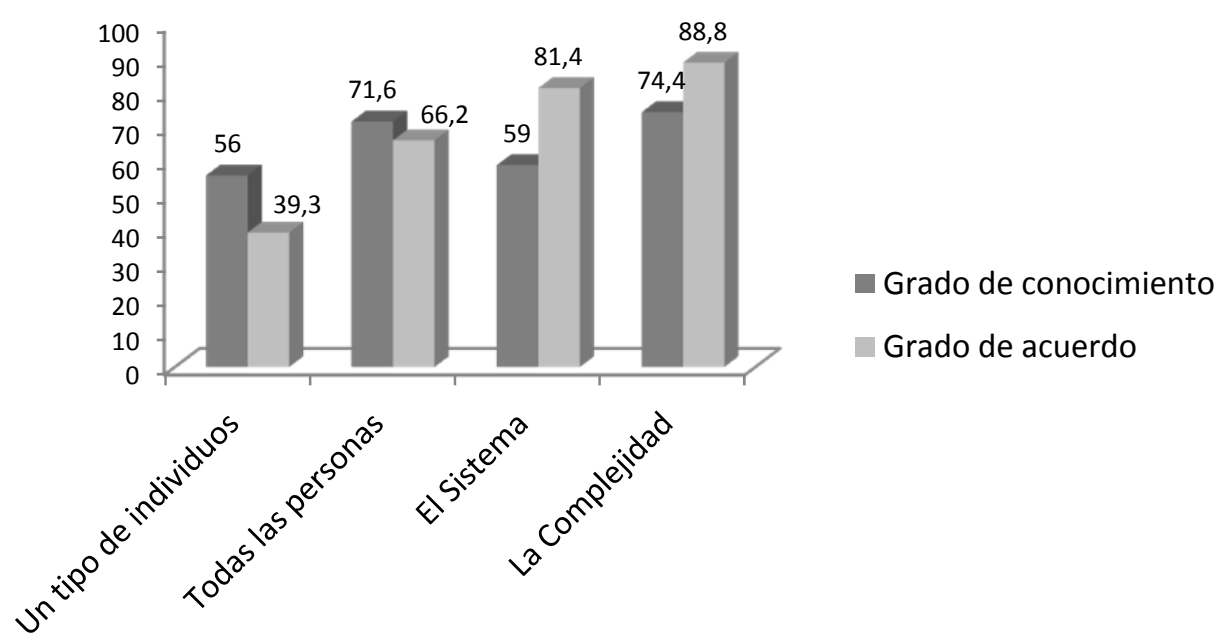

Gráfico 2 Comparación de grado de conocimiento/acuerdo con las teorías y enfoques de creatividad según el Modelo de tendencias en creatividad cCC'

Fuente: Elaboración propia

De las preguntas más pertinentes al objetivo que nos ocupa, indagamos acerca de cómo percibe la creatividad actual en la formación universitaria, en el sentido de como la comprende, y también como la aplica y experimenta en sí mismo.

De ello obtuvimos que en los enfoque de creatividad, destacan la mayor número de citas relevantes en la visión compleja de la creatividad como se parecía en el gráfico 3. 


\section{Tendencia en enfoques de creatividad (\%) n=50}

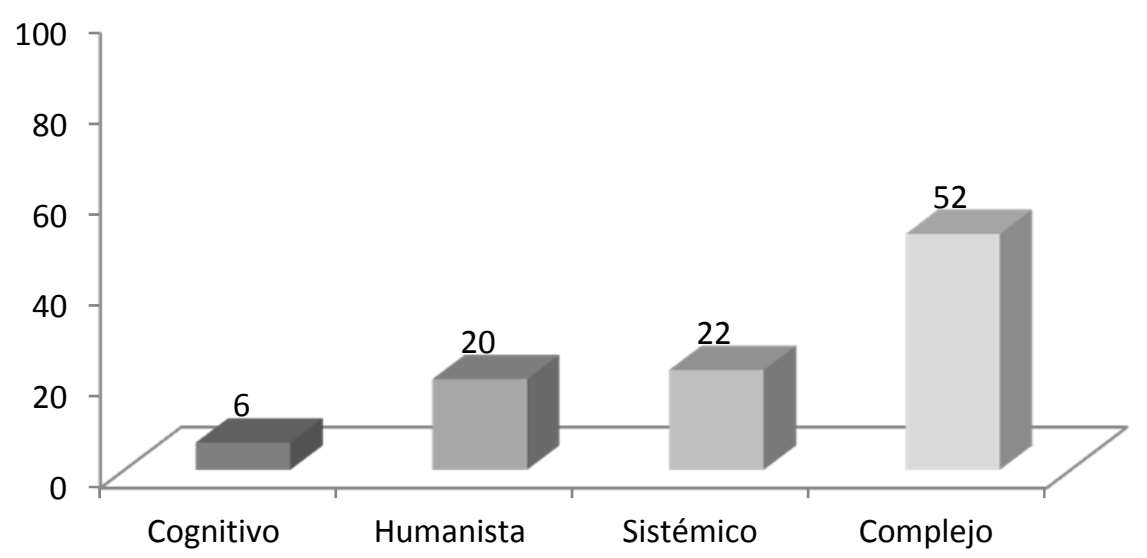

Gráfico 3 Gráfica de tendencia de enfoques en creatividad según todos las/os expertas/os.

Fuente: Elaboración propia

Por otra parte, esta tendencia se ha dado de manera más clara en aquellas citas de expertos que son docentes e investigadores en creatividad (y no sólo docentes o investigadores por separado) como puede observarse en el Gráfico 4.

\section{Tendencia en enfoques de creatividad según tipo de experto}

(\%) $\mathrm{N}=45$

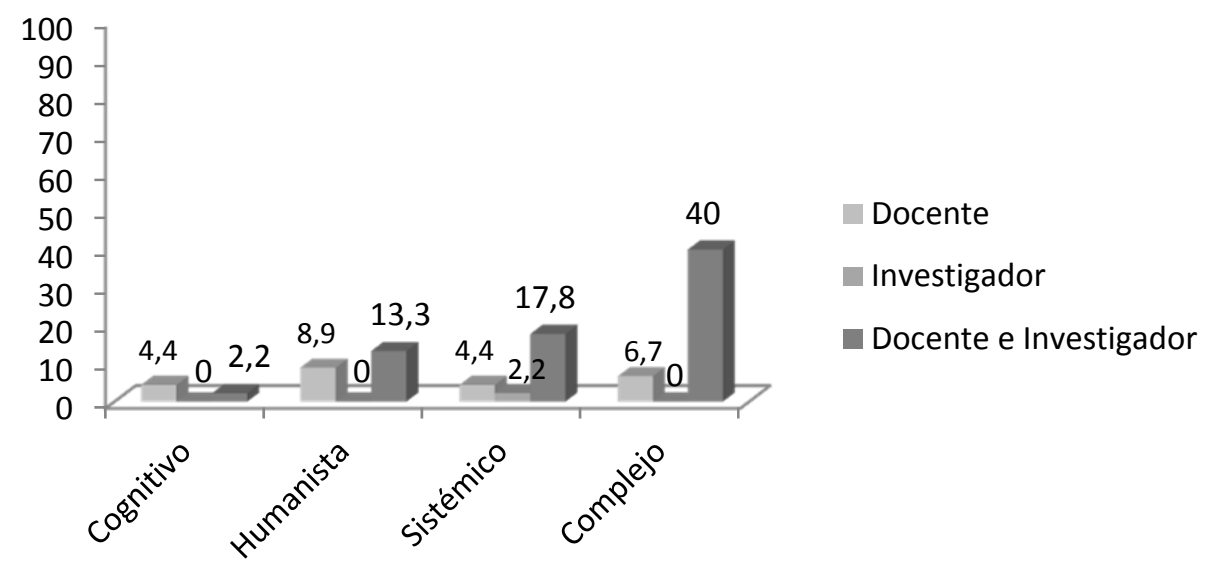

Gráfico 4. Tendencia en enfoques de creatividad según Tipo de experto.

Fuente: Elaboración propia 
En cuanto a la definición que realizan las/os expertas/os para la creatividad, se enmarca en los niveles crecientes de conciencia y complejidad desde lo humanista, hacia los sistémico y complejo. Se destaca de manera relevante, el nivel de la complejidad. Destacamos que estas citas se da con claridad en los docentes investigadores en creatividad, y de manera altamente relevante en las/os expertas/os del área de la Pedagogía como observamos en el Gráfico 5.

\section{Tendencia en creatividad según Área experto (\%) N=45}

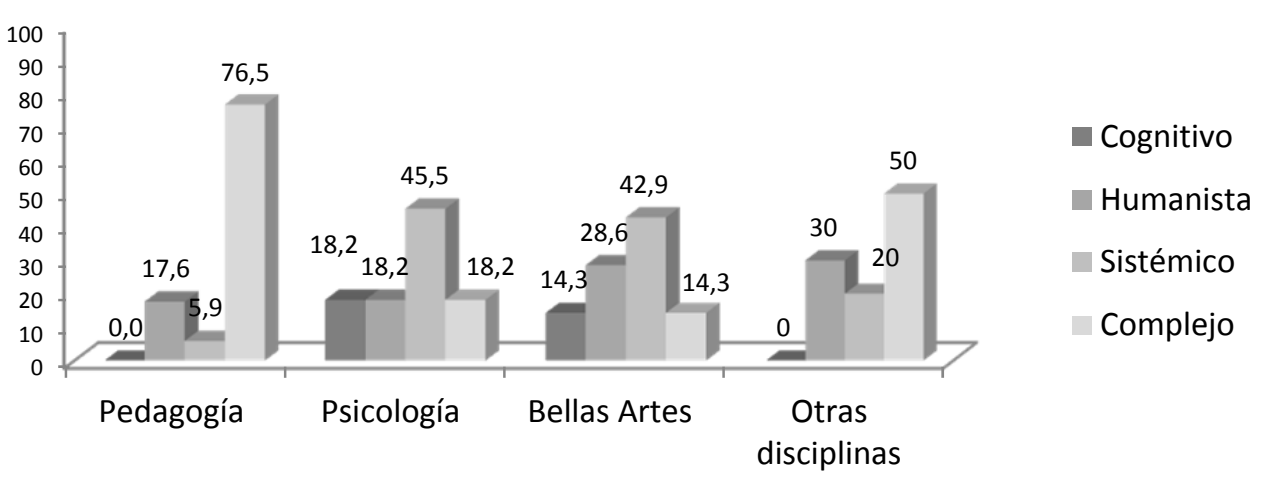

Gráfico 5. Tendencias en enfoques de creatividad según Área de expertas/os.

Fuente: Elaboración propia

En lo que respecta a los ámbitos de presencia futura de la creatividad en la formación universitaria podemos apreciar claramente como en la opinión y citas relevantes de los expertos, según el gráfico, la formación en creatividad debe estar orientada hace la integración sistémica y de mayor complejidad.

\section{Presencia Futura de la Creatividad (\%) N=45}

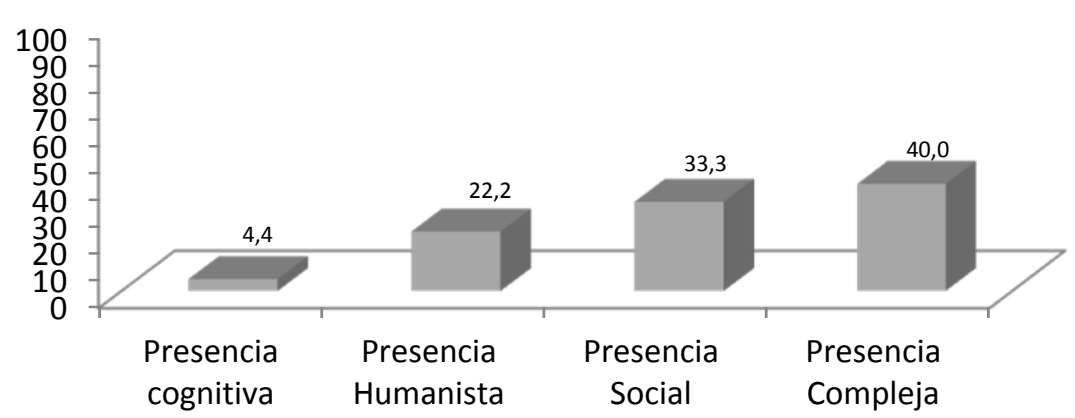

Gráfico 6 Ámbitos de presencia futura de la creatividad en la formación universitaria. 
Fuente: Elaboración propia

Como podemos observar, según la opinión de los expertos, la presencia de la creatividad en la formación universitaria futura tiene una clara tendencia hacia el ámbito de la complejidad. Lo hemos visto reflejado mayormente en las áreas de Pedagogía y Otras disciplinas, como en el caso de los docentes investigadores en creatividad.

En lo relativo al ámbito de interés por la investigación futura, se nos presenta asimismo una orientación hacia lo social y complejo. Eso lo podemos observar en general, y en todas las áreas de Pedagogía, Psicología, Bellas Artes y Otras disciplinas, así como claramente en Tipo de experto de los Docentes e investigadores en creatividad. Entendemos que los intereses por la investigación son variados, no obstante, observamos claramente que no se mencionan tanto el interés por la investigación hacia los procesos cognitivos como a una investigación en apertura al conocimiento en el ámbito social y de complejidad como podemos observar en el gráfico 7.

\section{Ámbito de interés de Investigación en creatividad (\%) n=45}

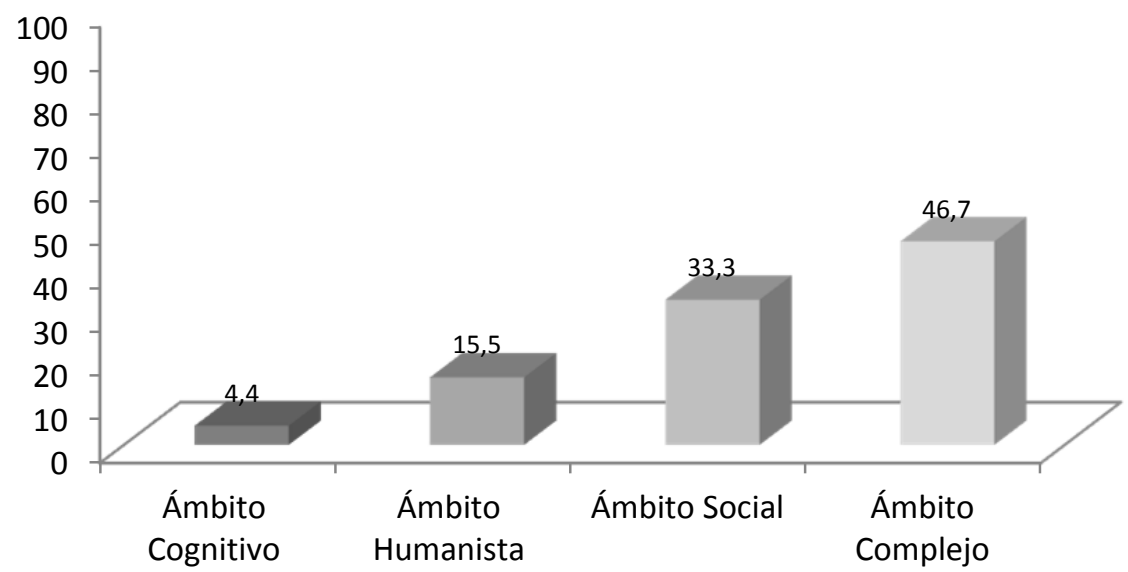

Gráfico 7. Gráfica de ámbito de interés en la investigación en creatividad.

Fuente: Elaboración propia

A manera de ejemplo, presentamos una selección de citas relevantes de esta cuestión (Figura 2), no obstante, fueron analizadas extensivamente y en rigor en todas las preguntas del cuestionario. 


\section{Código: Ámbito cognitivo}

\section{CASE: Case \#3}

El papel de las metáforas en la ciencia. La relevancia que tiene el pensamiento analógico en el avance científico.

CASE: Case \#11

Hay muchos, uno de ellos que me parece interesante, está basado en la cuestión neuronal y biológica del cerebro.

\section{Código: Ámbito humanista}

\section{CASE: Case \#7}

Lo que más me interesa es investigar las técnicas innovadoras de los profesores universitarios en su actividad educativa.

CASE: Case \#33

La creatividad en la universidad.

CASE: Case \#37

Didácticas creativas.

CASE: Case \#38

La resistencia de las personas a la creatividad.

\section{Código: Ámbito Social}

\section{CASE: Case \#1}

La dificultad para el reconocimiento de las personas creativas. Tantos y tantos creativos que se nos escapan, y tienen que morirse para que se les reconozca.

\section{CASE: Case \#2}

Creatividad como opuesto a la cultura hegemónica. Entender la creatividad desde el punto de vista cultural. La creatividad es una construcción de la mente occidental.

\section{CASE: Case \#25}

Las actitudes hacia el desempeño creativo en las diferentes culturas, dado que hay algunos idiomas antiguos que no la consideran.

\section{Código: Ámbito complejo}

\section{CASE: Case \#1}

La evolución es irreversible, pero no es gratuita. Estamos en la punta de la flecha, no somos la punta. Pero somos cada vez más seres que estamos en ello. Sabes que estás manejando la potencia y que eso es más potente que la operación producto. 
CASE: Case \#8

Liderazgo en la Unión Europea. Centro Europeo de Liderazgo Creativo.

CASE: Case \#9

Arte contemporáneo, creatividad, desarrollo personal y formación del profesorado.

CASE: Case \#13

El tema del campo energético y vibracional de la creatividad.

CASE: Case \#14

La espiritualidad. Los estudios de Osho, que debería ser leído por todos los maestros. Poder llegar a desarrollar todas las dimensiones del ser humano, todos sus talentos.

CASE: Case \#18

La conciencia, para mí, es un tema infinito. Que es la conciencia en creatividad, me parece que es una especie de círculo, latente, que se activa cuando se toman decisiones, y la creatividad es tomar decisiones.

CASE: Case \#21

La creatividad y los valores personales y sociales. También aplicados al ámbito de la espiritualidad, lo que significa ser vivo en el universo, y de cómo podemos hacer para hacer mejor.

Figura 2. Selección de citas relevantes según Ámbito de interés investigación futura en creatividad.

Fuente: Elaboración propia

El conjunto de resultados permite concluir que el conocimiento en creatividad avanza en mayores niveles de conciencia y complejidad, y que la forma de comprenderla, aplicarla y proyectarla hacia la formación y futuras investigaciones, se aproximan en este mismo sentido. Sin embargo, y a la luz de los resultados, además de ser un tema transversal de todas las dimensiones del ser humano y de la sociedad en su conjunto, aún queda mucho por hacer a nivel institucional, en la formación en creatividad y no en menor relevancia, para la autoformación de los propios docentes-investigadores en creatividad quienes aún se enfocan hacia la práctica creativa de su trabajo profesional, dejando en menor grado de interés el ámbito de su propia creatividad. Con todo ello, nos encontramos con un enorme interés por la profundización de su campo en materia de fundamentación científica, con el propósito de la mejora personal y social y de valores humanos que se vean ampliados en ámbitos espirituales y de mayor conciencia colectiva. 


\section{Conclusiones}

Las conclusiones del estudio referidas en los objetivos presentados, son las siguientes:

a) La aplicación de la creatividad en la formación aún es insuficiente. Se precisa de una mayor presencia en todos los ámbitos de las dimensiones humanas, así como una forma de potenciar una mayor responsabilidad social, cultural, ecológica.

b) Las expertos en creatividad vinculados tanto a la docencia como a la investigación tienen una mayor responsabilidad en ampliar el campo de aplicación, puesto que son ellos los que, desde su conocimiento, muestran una mayor tendencia hacia mayores niveles de conciencia y complejidad aplicada a este campo.

c) Se constata que hay una demanda y una orientación de enfoques hacia una consideración compleja y transdisciplinar de la creatividad, que incluiría en el conocimiento y su ámbito la mejora de la formación y las implicaciones éticas de la creatividad. Los datos del estudio transdisciplinar pueden contribuir a ampliar la discusión científica en el ámbito formativo universitario, y más específicamente a la formación en creatividad hacia nuevas o mejores formas de hacer, ser y convivir creativa y conscientemente.

d) Tanto la transdisciplinariedad como el enfoque de complejidad evolutiva son potentes referentes epistemológicos, metodológicos y prácticos para las múltiples aplicaciones de la creatividad, en especial, en la aplicación social y de contextos de innovación didáctica.

e) Es preciso continuar investigando la creatividad con enfoque transdisciplinar y de complejidad evolutiva para todo cuanto beneficie a programas de formación en general. Específicamente, para promover de este modo cambios hacia la mejora social y ecológica en un contexto de libertad, virtudes y valores humanos, fraternidad y conocimiento compartido. Con esta perspectiva epistemológica parece posible en mayor medida aspirar a formar e investigar en una creatividad con base en la conciencia donde la ética, los valores y las virtudes humanas puedan participar de un modo más activo del desarrollo profesional de los docentes y donde cada persona pueda reconocer, valorar y compartir su potencial creativo, para el beneficio del entorno social y ecosistémico cercano y mediato de los que forma parte.

\section{Referencias bibliográficas}

ALFONSO, V. (2006). Creatividad en la psicología. Creatividad como fenómeno complejo y multidimensional, en S. de la Torre y V. Violant (coord.), Comprender y Evaluar la Creatividad, Vol. I, 323-333. Málaga: Aljibe.

ALMENDRO, M. (2006). La consciencia transpersonal. Barcelona: Kairós.

AMABILE, T.M. (1983). The Social Psychology of Creativity. New York: Sprenger Verlag.

BLAY, A. (1980). Ser. Psicología de la autorrealización. Madrid: Índigo. 
BODEN, M. (1994). La mente creativa. Mitos y mecanismos. Barcelona: Gedisa.

BONO, E. de (1977). Lateral Thinking. Harmondsworth (England): Penguin Books.

CABRERA, J. (2008). Tendencias en enfoques sobre creatividad humana. Una mirada evolutiva”. En A. de la Herrán y J. Paredes (coords.), Didáctica General. La práctica de la enseñanza en Educación Infantil, Primaria y Secundaria. Madrid: Mc Graw-Hill-Interamericana.

CABRERA, J. (2009). Creatividad Hoy. Una Evolución hacia Mayores Niveles de Conciencia y Complejidad. Educación y Futuro (21), 15-42.

CABRERA, J. (2010). Transdisciplinariedad en la formación universitaria. Una investigación en curso, en Torre y Pujol (coord.), Creatividad e Innovación. Enseñar e investigar con otra conciencia, 177-191. Madrid: Universitas.

CABRERA, J. (2011). Creatividad, conciencia y complejidad: Una contribución a la epistemología de la creatividad para la formación. Tesis doctoral. Facultad de Formación de Profesorado y Educación. Universidad Autónoma de Madrid. Madrid.

CABRERA, J. (2012). Creatividad y Complejidad en la Formación Universitaria. Tendencias desde una Perspectiva Docente. Revista Matices del Posgrado Aragón, 31-66.

CSIKSZENTMIHALYI, M. (1996). Creativity: Flow and the Psychology of Discovery and Invention. New York: Harper Perennial.

FERRER, J. N. (2003). Espiritualidad creativa. Una visión participativa de lo transpersonal. Barcelona: Kairós.

FREUD, S. (1923). Ego and the Id. New York: Norton.

GONZÁLEZ-GARZA, A.M. (2006). El desarrollo de la consciencia desde la óptica transpersonal, en M. Almendro, La consciencia transpersonal, 315-351. Barcelona: Kairós.

GUILFORD, J. P. (1950). Creativity. The American Psychologist, 5 (9), 444-454.

HASHIMOTO, E. (2013). Un enfoque metodológico alternativo para investigar en educación. Tesis doctoral. Facultad de Formación de Profesorado y Educación. Universidad Autónoma de Madrid. Madrid.

HERNÁNDEZ SAMPIERI, R., Fernández Collado, C. y Baptista Lucio, P. (2003). Metodología de la investigación. México: Mc Graw Hill Interamericana.

HERRÁN, A. de la (1998). Cómo estudiar en la universidad. Madrid: Universitas.

HERRÁN, A. de la (2003). El Nuevo Paradigma Complejo-Evolucionista. Revista Complutense de Educación, 14 (2), 499-562.

HERRÁN, A. de la (2005). El nuevo paradigma complejo evolucionista en educación. En A. de la Herrán, E. Hashimoto y A. Machado, Investigar en educación. Fundamento, aplicación y nuevas perspectivas, 481-571. Madrid: Dilex. 
HERRÁN, A. de la (2008). Didáctica de la creatividad. En A. de la Herrán y J. Paredes (coords.), Didáctica General. La práctica de la enseñanza en Educación Infantil, Primaria y Secundaria, 151-176. Madrid: Mc Graw Hill.

HERRÁN, A. de la (2009a) Contribución al Concepto de Creatividad: Un Enfoque Paquidérmico ( $1^{\text {a }}$ parte), Educación y Futuro. Revista de Investigación Aplicada y Experiencias Educativas (21), 43-70.

HERRÁN, A. de la (2009b). Contribución al Concepto de Creatividad: Un Enfoque Paquidérmico ( $2^{\mathrm{a}}$ parte). Educación y Futuro. Revista de Investigación Aplicada y Experiencias Educativas (22), 151-175.

HERRÁN, A. de la (2010) Contribución al Concepto de Creatividad: Un Enfoque Paquidérmico ( $3^{\mathrm{a}}$ parte). Educación y Futuro. Revista de Investigación Aplicada y Experiencias Educativas (23), 129-162.

HERRÁN, A. de la (2011a). Complejidad y Transdisciplinariedad. Revista Educação Skepsis, 1 (2), 294-320.

HERRÁN, A. de la (2011b). Reflexiones para una Reforma Profunda de la Educación desde un Enfoque Basado en la Complejidad, la Universalidad y la Conciencia. Educación XX1 (14), 245-264.

HERRÁN, A. de la (2013). ¿Una transdisciplinariedad inmadura? Consideraciones críticas radicales (pensando desde la Pedagogía y la Didáctica). En J. Paredes, F. Hernández, y J.M. Correa (Eds.). La relación pedagógica en la universidad, lo transdisciplinar y los estudiantes. Desdibujando fronteras, buscando puntos de encuentro (pp. 30-51). Madrid: Universidad Autónoma de Madrid. http://hdl.handle.net/10486/13152 (Consultado 4 de junio de 2014).

HERRÁN, A. de la (2014). Enfoque radical e inclusivo de la formación. REICE. Revista Iberoamericana sobre Calidad, Eficacia y Cambio en Educación, 12(2). Recuperado de: http://www.rinace.net/reice/numeros/arts/vol12num2/art8.pdf (Consultado 4 de julio de 2014).

KRIS, E. (1952). Psychoanalytic exploration in art. New York: International University Press.

KUBIE, L.S. (1958). Neurotic distortion of the creative process. Kansas: University of Kansas.

LANDAU, E. (2002). El vivir creativo. Teoría y práctica de la creatividad. Barcelona: Herder.

López PÉREZ, R. (2006). Diccionario de la creatividad. Conceptos y expresiones habituales del campo temático de la creatividad. Santiago de Chile: Universidad Central. Chile.

MAC KINNON, D. W. (1975). IPAR's contribution to the conceptualization and study of creativity, in I. A. Taylor \& J. W. Getzels (Eds.), Perspectives in creativity, 60-89. Chicago: Aldine. 
MASLOW, A. (1985). La personalidad creadora. Barcelona: Kairós.

MATURANA, H. (1997). Emociones y lenguaje en educación y política. Santiago de Chile: Dolmen.

MC MILLAN, J. y SCHUMACHER, S. (2005). Investigación Educativa. Madrid: Pearson.

MOONEY, C. M. (1957). Age in the development of closure ability in children. Canadian Journal of Psychology, 11, 216-226.

MORAES, M. C. (2007). Complejidad, transdisciplinariedad y educación: Algunas reflexiones. Encuentros Multidisciplinares, 9 (25), 4-13.

MORIN, E. (2003). Los siete saberes necesarios para una educación de futuro. Barcelona: Paidós.

MORIN, E. (2004). Introducción al pensamiento complejo. Barcelona: Gedisa.

MORIN, E., CIURANA, R. y MOTTA, R. (2003). Educar na era planetária: O pensamento como método de aprendizagem pelo erro e incerteza humana. São Paulo: Cortez Editora-UNESCO.

ROGERS, C. (1980). El camino del ser. Barcelona: Kairós.

ROMO, M. (1997). Psicología de la creatividad. Barcelona: Paidós.

RUBIA VILA, F. (2007). La conexión divina. La experiencia mística y la neurobiología. Barcelona: Crítica.

RUNKO, M. (2007). Creativity y theories and dreams: research, development, and practice. Boston: Elsevier Academic Press.

SÁNCHEZ-CRIADO, T. y BLANCO, F. (2005). Herramientas Básicas para la Investigación Cualitativa. Introducción al manejo del programa de análisis QDA Miner 1.2. Manuscrito. Madrid.

STERNBERG, R. (1977). Intelligence, information processing,and analogical reasoning: The componential analysis of human abilities. N. J.: Erlbaum.

TORRE, S. de la (2006). “Teoría interactiva y psicosocial de la creatividad”, en S. de la Torre y V. Violant (Coords.), Comprender y evaluar la Creatividad. Vol. I, 123154. Málaga: Aljibe.

TORRE, S. de la, PUJOL, M. y SANZ, G. (2007). Transdisciplinariedad y ecoformación. Una nueva mirada sobre la educación. Barcelona: Universitas.

TORRE, S. de la, y MORAES, M. C. (2006). Investigar en creatividad bajo el pensamiento complejo, en S. de la Torre y V. Violant, Comprender y evaluar la creatividad. Vol. 2, 33-72. Málaga: Aljibe.

TORRE, S. de la, y MORAES, M. C. (2008). "Decálogo del docente transdisciplinar y creativo”. Red Internacional de Escuelas Creativas, 152-153.

WILBER, K. (2005). El espectro de la conciencia. Barcelona: Kairós. 


\section{Correspondencia con los autores}

Jessica CABRERA CUEVAS

Dpto. Didáctica y Teoría de la Educación (I-315)

Facultad de Formación de Profesorado y Educación.

Universidad Autónoma de Madrid

C/ Francisco Tomás y Valiente, $n^{\circ} 3$

28049 Madrid

e-mail: jessica.cabrera@uam.es

Agustín de la HERRÁN GASCÓN

Dpto. Didáctica y Teoría de la Educación (I-315)

Facultad de Formación de Profesorado y Educación.

Universidad Autónoma de Madrid

C/ Francisco Tomás y Valiente, $n^{\circ} 3$

28049 Madrid

e-mail: agustin.delaherran@uam.es 